Dilution mistakes, overdoses, side effects in the era of the pandemic

Pardis Tabaee Damavandi

No conflicts of interest to disclose 
Dilution mistakes, overdoses, side effects in the era of the pandemic

Dilution errors are a common nursing mistake that are linked to fatigue, over-work, lack of experience or supervision, health issues, disattention and over-confidence. When dilution mistakes are made whereby a dilution is missed or the product that needs to be inoculated systemically or nonetheless promptly within the patient, then risks become great. The risks are obviously related to the manifestation of an adverse reaction to the medicinal product being administered, such as a drug in its more variate dosage forms, and particularly in the administration of the vaccine, which is what we have experienced during the coronavirus pandemic. It is true that an excessive dilution reduces the potency and effectiveness of the medicinal product (vaccine included), hence why the most experienced nurses tend to dilute everything, even when not required, because it is safer for the patients, but some medicinal products do not require dilution and can be left concentrated, this can lead to mistakes, such as forgetting to dilute, not diluting enough, and thus resulting to overdoses, that can as a result provoke important side effects. This is particularly important in the case of dosage forms, such as intravenous and intramuscular that, as we know, have more "immediate" rapid profiles and pharmacokinetics, compared to oral dosage forms for instance, thus the dosage form chosen during the design of vaccines should also be taken into account, especially if the toxicological LD50/ED50 interval values are narrow, making the active principle ingredient toxic. This has been the case, with some mRNA vaccines, that have had multiple dose adjustments during the first phase trials, nevertheless, adverse events, of a serious nature as well, where no morbidity has been observed, but where dangerous risks have been attributed to the overdoses have resulted. One particular case of side effects were the pericarditis, and myocarditis adverse reactions of one of the "safest mRNA" vaccine which undiluted, has led to the penetration of the pericardial sac or the myocardium of the vaccine powder (suspended), provoking an 
immune response cascade to remove the infiltrations, that out-turned to the above-mentioned risky and painful cardiac inflammatory response (potentially culminating into a CHD).

\section{Overdose of vaccines}

Overdose of certain vaccines have resulted without appropriate mixture or dilution of the suspended powders, which have caused several overdoses and adverse responses in the patients. The problem with this is that many of these overdoses are undetected, because they are under-reported by the practitioner, being that nurses do not always realise the drug has not been administered properly, but vaccines that are generally inoculated intramuscularly do pose a moderate risk for reactions, not as high as intravenous injections, but quite fast, compared to other administration routes, it is therefore important to pay attention and to be supervised, at least, in the step of preparation of the vaccine that need to be inoculated. Another way to prevent side effects is to associate side effects to the batch administered. If only some patients have experienced side effects from one batch, then the mistake may be identified in the operator, particularly if the side effects are not attributable to the batch or vaccine itself, because multiple inoculations in the same patients have given rise to diverse reactions. The diverse reactions are caused by how concentrated or diluted the vaccine were, so a focus on dilution and dissolution in solutions to be administered or reduction of the number of suspended powders must be taken into account, if another administration route can't be taken into account.

\section{Dilution mistakes in other scenarios}

Several reports exist whereby the hospital staff that had made dilution errors wasn't confident with the preparation of diluted preparations because that role is usually covered by pharmacists or because training on dilutions was not crucial during the formative years. Not all hospitals have a pharmacist, as the pharmacist-to-nurse ratio is lower. Likewise, the doctor-to nurse ratio is lower too. It is therefore important to provide supervision in the drug administration programmes by both the medical and pharmaceutical staff, at least during the drug preparation 
stage, because delivery errors aren't as prominent in the nursing staff than dilution errors. The nursing staff is very skilled in injectable preparation delivery, but most reports pertain dilution errors. Dilution errors that turn out to be fatal for the patient, have an enormous impact on the nurse's wellbeing too, as it happened in several cases too. It is particularly important to be careful with overdoses, nevertheless accidental overdoses can be carried out by patients too, and thus communication between professionals and between professionals and patients, with enough supervision should always be present. 